\title{
Wing structure and neural encoding jointly determine sensing strategies in insect flight
}

\author{
Alison I. Weber $\oplus^{*}$, Thomas L. Daniel $\odot$, Bingni W. Brunton $\odot$ \\ Department of Biology, University of Washington, Seattle, Washington, United States of America \\ *aiweber@uw.edu
}

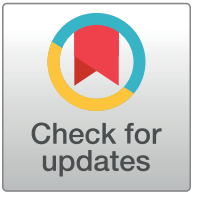

\section{G openaccess}

Citation: Weber Al, Daniel TL, Brunton BW (2021) Wing structure and neural encoding jointly determine sensing strategies in insect flight. PLoS Comput Biol 17(8): e1009195. https://doi.org/ 10.1371/journal.pcbi.1009195

Editor: Barbara Webb, The University of Edinburgh, UNITED KINGDOM

Received: January 22, 2021

Accepted: June 18, 2021

Published: August 11, 2021

Peer Review History: PLOS recognizes the benefits of transparency in the peer review process; therefore, we enable the publication of all of the content of peer review and author responses alongside final, published articles. The editorial history of this article is available here: https://doi.org/10.1371/journal.pcbi.1009195

Copyright: @ 2021 Weber et al. This is an open access article distributed under the terms of the Creative Commons Attribution License, which permits unrestricted use, distribution, and reproduction in any medium, provided the original author and source are credited.

Data Availability Statement: Code that reproduces all simulation data can be found at https://github. com/aiweber/optimal_sensing_ELwing.

Funding: This work was supported by the Washington Research Foundation (AIW); the

\section{Abstract}

Animals rely on sensory feedback to generate accurate, reliable movements. In many flying insects, strain-sensitive neurons on the wings provide rapid feedback that is critical for stable flight control. While the impacts of wing structure on aerodynamic performance have been widely studied, the impacts of wing structure on sensing are largely unexplored. In this paper, we show how the structural properties of the wing and encoding by mechanosensory neurons interact to jointly determine optimal sensing strategies and performance. Specifically, we examine how neural sensors can be placed effectively on a flapping wing to detect body rotation about different axes, using a computational wing model with varying flexural stiffness. A small set of mechanosensors, conveying strain information at key locations with a single action potential per wingbeat, enable accurate detection of body rotation. Optimal sensor locations are concentrated at either the wing base or the wing tip, and they transition sharply as a function of both wing stiffness and neural threshold. Moreover, the sensing strategy and performance is robust to both external disturbances and sensor loss. Typically, only five sensors are needed to achieve near-peak accuracy, with a single sensor often providing accuracy well above chance. Our results show that small-amplitude, dynamic signals can be extracted efficiently with spatially and temporally sparse sensors in the context of flight. The demonstrated interaction of wing structure and neural encoding properties points to the importance of understanding each in the context of their joint evolution.

\section{Author summary}

In addition to generating forces for flight, insect wings also serve an important role as sensory structures, providing rapid feedback about wing bending that is used to stabilize flight. While much is known about how wing structure affects aerodynamic performance, the effects of wing structure on sensing remain unexplored. Using a computational model of a flapping wing, we examine how sensing strategies depend on wing stiffness and sensor properties. We show that body rotations can be accurately detected with a small number of sensors on the wing across a wide range of conditions. Optimal sensor locations are clustered at either the wing base or wing tip, depending on a combination of wing stiffness and sensor properties. Moreover, sensing performance is robust to multiple kinds of 
eScience Institute at the University of Washington (AIW); and the Air Force Office of Scientific Research awards FA9550-18-1-0114 (BWB) and FA9550-19-1-0386 (BWB, TLD). The funders had no role in study design, data collection and analysis, decision to publish, or preparation of the manuscript.

Competing interests: The authors have declared that no competing interests exist. perturbations. Our work provides a basis for understanding how wing structure impacts incoming sensory information during flight.

\section{Introduction}

The physical structure of an animal's body transforms the incoming sensory information and can either facilitate or constrain sensing capacity. Indeed, body parts in many systems serve to preprocess sensory inputs in ways that are beneficial for the organism, extracting relevant features and reducing downstream computational burdens. For instance, the decrease in stiffness from the base to apex of the mammalian cochlea promotes frequency selectivity along its length [1], and the response properties of mechanosensors that encode high-frequency vibrations in mammalian skin are determined largely by the viscoelastic properties of layered, fluidfilled capsule surrounding nerve endings [2]. In systems that rely on mechanosensation in particular, there is a large body of work pointing to the importance of structure in preprocessing sensory inputs (reviewed in [3]). On the other hand, there may be structural limits that constrain the numbers and locations of sensory receptors. In the vibrissal system, for example, mechanosensory neurons are located only at the whisker base, so information about an object's point of contact with the whisker cannot be directly measured [4]. Thus, the physical properties of non-neural structures play an important role in determining how stimuli are experienced and transduced by sensory receptors embedded in the body.

Sensory receptors transduce stimuli into electrical signals, typically taking the form of action potentials, in which the incoming signal is converted into a series of all-or-none events. Sensory neurons respond selectively to particular features of the stimulus; for instance, auditory neurons in the cochlea respond to particular frequencies, and visual neurons in the retina respond to distinct temporal patterns of illumination [5-7]. This transformation from input to response can often be accurately represented by a two-part model of neural encoding $[8,9]$. One part consists of a stimulus feature or features to which the neuron is sensitive, and the second part is a nonlinear function that captures how selective the neuron is for those features. Importantly, neural encoding properties define the information available to the rest of the nervous system about the animal's environment and its own body. These properties may adapt across multiple timescales, changing over rapid timescales based on the history of inputs as well as over evolutionary time $[10,11]$.

In this paper, we focus on the interaction of body structure and sensory encoding properties in the context of insect flight control, where wings provide rapid sensory feedback necessary for stable flight [12-16]. Although extensive previous work has examined how wing structure impacts aerodynamic performance (for a few examples, see [17-22]), the impacts of wing structure on sensing remain unexplored. Structural properties (e.g., geometry, flexural stiffness) interact with forces acting on the wing to produce local spatiotemporal patterns of strain that are sensed by the nervous system. Local strain is encoded by sensors, called campaniform sensilla, distributed sparsely over the wings at consistent locations across individuals of a given species. It has recently been demonstrated that only a small number of sensors are needed to read out behaviorally relevant information about body rotations, with neural sensors providing advantages over sensors that directly encode strain [23]. Sparse sensing strategies have advantages in terms of both energetic cost and robustness [24-27]. However, no previous work has examined the impact of wing structure on sparse sensing strategies.

In this paper, we show that just a few spiking sensors can detect subtle differences in strain that arise from the relatively tiny forces produced by body rotation compared to wing flapping. 
We use a simple model, with wing size and stiffness based on the wings of Manduca sexta, to characterize spatiotemporal patterns of strain over a wing during flapping. We then encode this strain in a population of spiking sensors, which we refer to as neural-inspired sensors, whose response properties are based on experimental measurements from mechanosensory neurons. With a sparsity-promoting optimization method, we solve for the locations of a small, fixed number of sensors for detecting body rotation about different axes. We focus on systematically varying wing stiffness because it is an important structural property that varies widely across species [28], within species [29], and even over an animal's lifetime [30]. We demonstrate that wing stiffness and neural encoding properties jointly determine the accuracy of body rotation detection and optimal sensor locations. Moreover, sensing performance is remarkably resilient to sensor loss and external disturbances, suggesting that sparse placement of sensors can be used for efficient, robust sensing in the context of flight.

\section{Results}

To understand how sensing strategies and performance are impacted by wing structure, we focus on one specific sensing goal: finding a minimal set of strain-encoding, spiking mechanosensors on the wing that are effective in detecting body rotation. Next, given the timing of a single spike from each of a set of sensors placed at optimal locations, we ask how well rotation of the body about different axes can be discriminated. We then analyze this performance and the optimal locations of the sensors on the wings, assessing how they change as we manipulate wing stiffness and neural encoding properties.

We first simulate spatiotemporal patterns of strain over a flapping wing using an EulerLagrange model inspired by the wings of the hawkmoth Manduca sexta [31] (Fig 1A, top). The dimensions, flexural stiffness, and flapping frequency are based on previously measured quantities [29]. Strain data are acquired for two conditions: one in which the wing is flapping, and another in which the body is undergoing rotation while the wing is flapping. The time history of strain is then encoded by a population of neural-inspired sensors, whose encoding properties are based on experimental measurements of mechanosensors in hawkmoth wings [14]. The output of this neural encoding step is a pattern of spikes, temporally sparse all-or-none signals (Fig 1A, middle). We then solve for a spatially sparse set of sensors (10 sensors, unless otherwise noted) that can be used to detect body rotation based on spike timing (Fig 1A, bottom). A single spike time from each sensor in this subpopulation is used to determine whether or not the wing is undergoing rotation during each wingbeat. We repeat this procedure for wings of different stiffness (Fig 1B, top) and sensors with different thresholds (Fig 1B, bottom).

\section{Spike timing and precision determine local peaks in accuracy as a function of wing stiffness}

To examine how sparse sensing strategies depend on wing structure, we first vary wing stiffness while holding neural encoding properties constant (linear filter frequency parameter $\omega=$ 1 ; neural threshold $\beta=0.2$ ). The range of wing stiffness values examined centers on the average reported flexural stiffness for hawkmoth forewings (Methods) [28, 29]. We solve for sparse sensor locations that can detect rotation and evaluate performance using the 10 best sensors.

We begin by focusing on rotation in the yaw axis and later turn to pitch and roll. Classification accuracy changes nonmonotonically with wing stiffness, peaking near $100 \%$ accuracy at a wing stiffness similar to that for hawkmoth wings (stiffness factor $=1$ ). There is a second, smaller peak of approximately $75 \%$ accuracy at a much lower stiffness (Fig $2 \mathrm{~A}$ ). In both cases, the optimal sensor locations occur at the wing tip. The single best sensor location for each of 
A
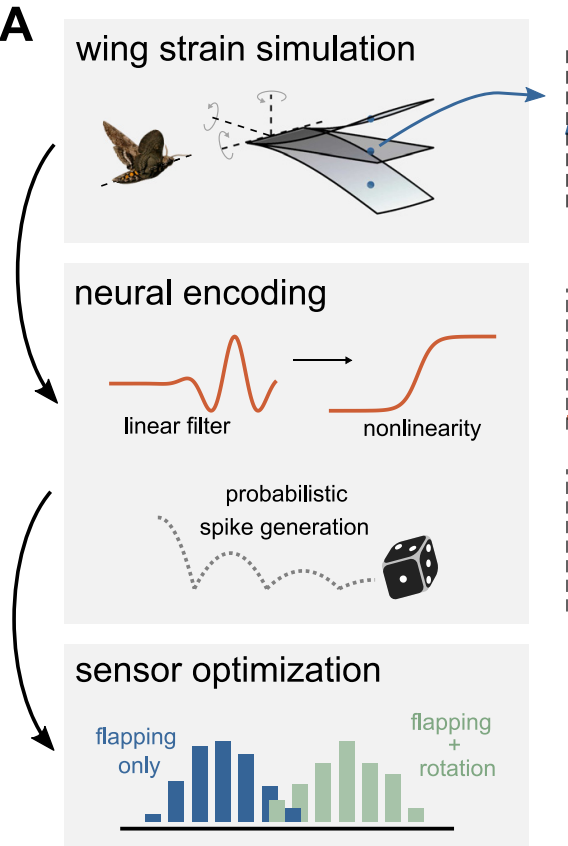

one wingbeat $(40 \mathrm{~ms})$
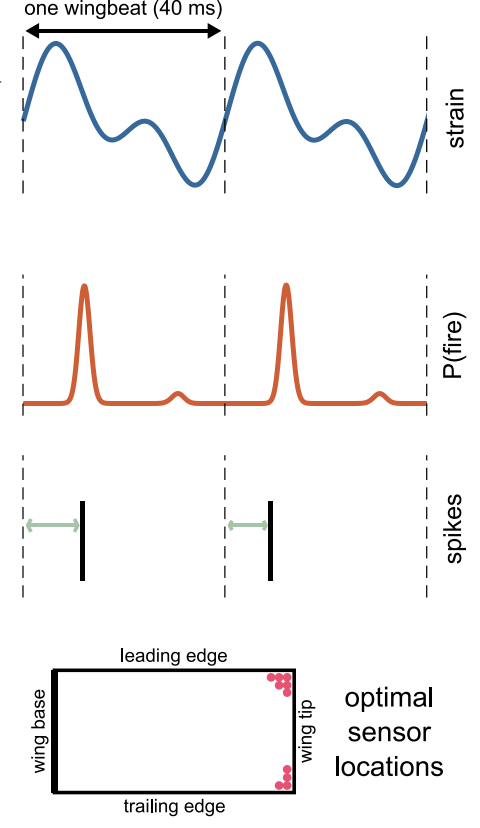

B

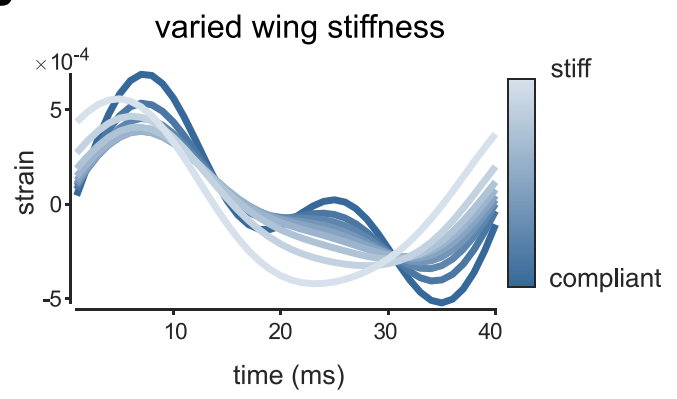

varied neural threshold

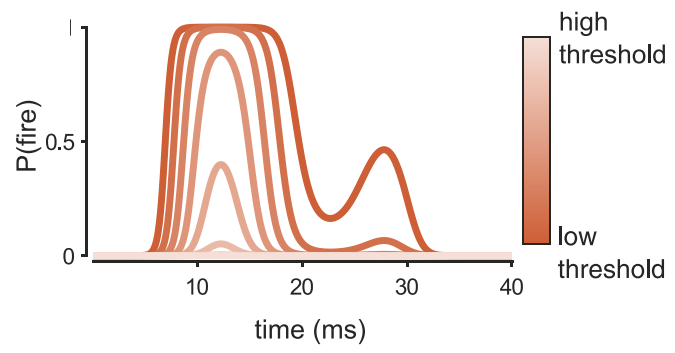

Fig 1. Wing stiffness and neural encoding properties determine optimal sensor locations on a flapping wing. A: Top: Strain over time is simulated at each possible sensor location in an Euler-Lagrange model of a flapping wing [23, 31]. The wing may be subject to rotation in different axes. Strain is shown for an example sensor location (blue dot). Middle: Strain at each location is encoded by a neural-inspired sensor. Strain is convolved with a linear filter and passed through a static nonlinearity to generate a probability of firing a spike over time, $\mathrm{P}$ (fire). Spikes are probabilistically generated, so that exact spike time varies from wingbeat to wingbeat. Bottom: The full data set, a matrix of spike times at all sensor locations for each wingbeat, is used to find a subset of sensors from which information about rotation can be read out. Linear discriminant analysis is used to find the vector $w$, such that when the data are projected onto $w$ the means of the flapping only and flapping with rotation data are maximally separated. B: Top: Changing wing stiffness produces different strain (shown for identical location) over the course of each wingbeat. Bottom: Changing the threshold of the neural encoding nonlinearity alters of the probability of firing over the course of the wingbeat, with higher thresholds resulting in lower probability. Hawkmoth image in A courtesy of Armin J Hinterwirth.

https://doi.org/10.1371/journal.pcbi.1009195.g001

these stiffness values is shown in Fig 2A, although all of the 10 best sensors in each case fall at the wing tip and show similar patterns of strain and spiking over time.

Differences in spike timing and spike timing precision underlie these local peaks in accuracy. For more compliant wings, the filtered strain in optimal sensors quickly crosses threshold (Fig 2B, left), leading to sharp transitions from zero probability of firing ( $\mathrm{P}($ fire $)$ ) a spike to certainty of firing a spike (P(fire) $\sim 1$, Fig $2 \mathrm{C}$, left). This results in precisely timed spikes both when the wing is flapping only and flapping with rotation (Fig $2 \mathrm{D}$ and $2 \mathrm{E}$, left). Small differences in filtered strain under these two conditions translates to small but detectable differences in spike timing. With as few as 10 sensors, wing rotation can be detected with $\sim 75 \%$ accuracy.

For stiffer wings, the filtered strain in optimal sensors varies less over time, barely reaching threshold (Fig 2B, right). Thus, there is an overall lower probability of firing, but the difference between flapping only and flapping with rotation is amplified: small differences in filtered strain translate to clear differences in $\mathrm{P}$ (fire) over time (Fig $2 \mathrm{C}$, right). This change in $\mathrm{P}$ (fire) is reflected in spike timing, with the first spike being much more likely to occur earlier in the wingbeat cycle in the flapping case (around $5 \mathrm{~ms}$ ) compared to flapping with rotation (around $25 \mathrm{~ms}$; Fig 2D and 2E, right). Lower precision in spike timing is offset by large differences in the time to the first spike, resulting in a classification accuracy approaching $100 \%$.

These results suggest that classification performance for a given wing stiffness might be improved by altering the neural threshold. We next examine how neural encoding properties 
A

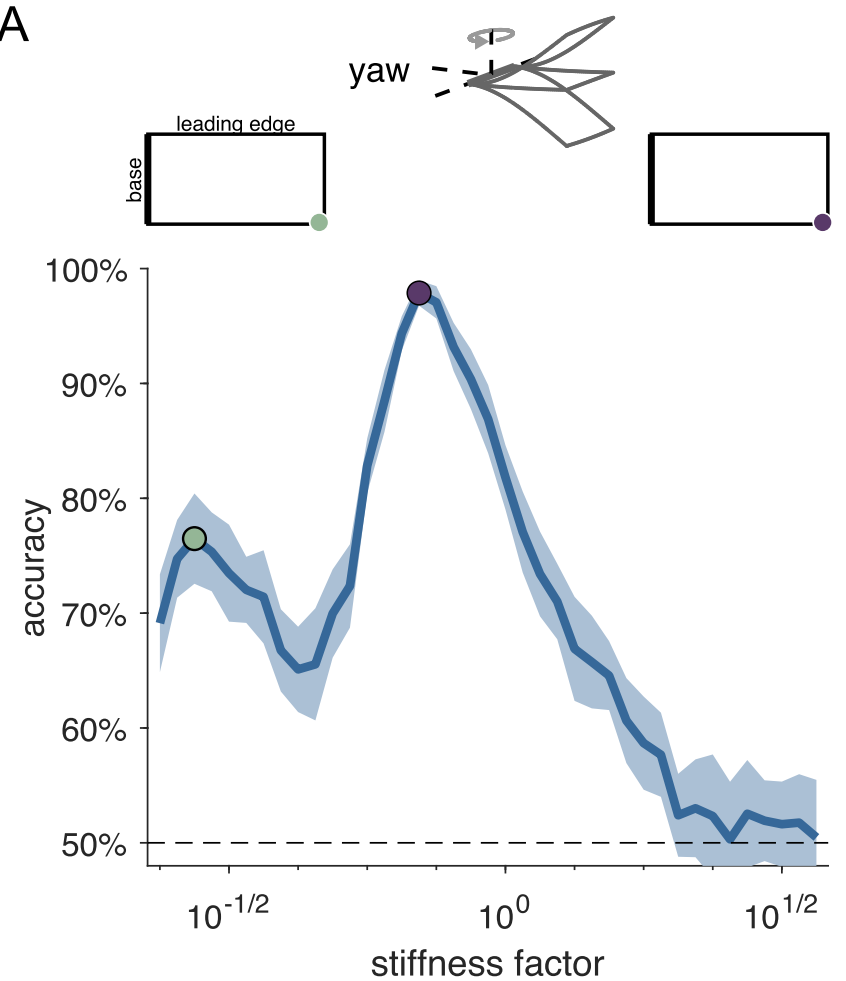

$\mathrm{B}$

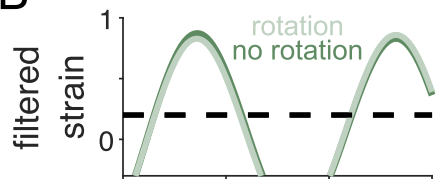

C

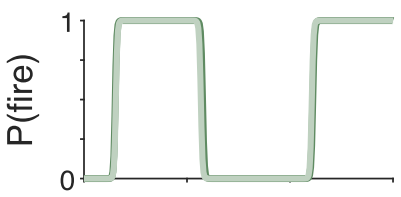

$\mathrm{D}$

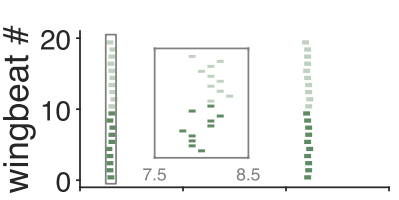

$\mathrm{E}$

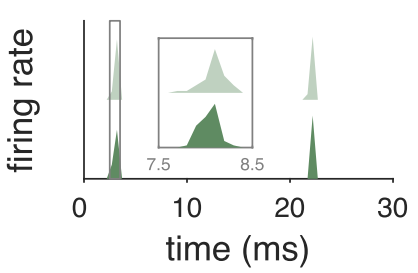

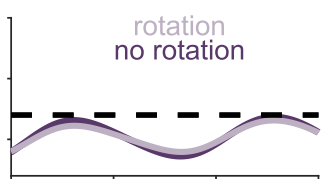
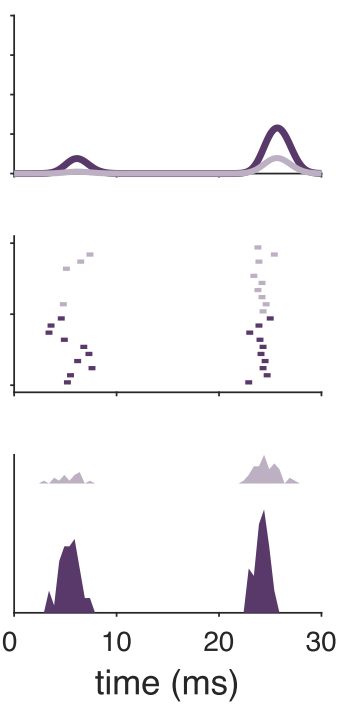

Fig 2. Wing stiffness interacts with neural threshold to determine spike timing. A: Accuracy as a function of wing stiffness. Chance accuracy is $50 \%$, and stiffness factor 1 corresponds to $3 \mathrm{GPa}$, comparable to experimentally measured stiffness in the hawkmoth [28]. Green and purple dots indicate local peaks in accuracy. Shaded area indicates \pm 1 standard deviation. Top: Center schematic indicates the direction of rotation. Left and right schematics show the location of the single best sensor for each stiffness value. B: Filtered strain over a single wingbeat for flapping only ("no rotation") and flapping with rotation ("rotation") conditions at a single sensor location. Green and purple sensor locations in A correspond to left/green and right/purple filtered strain. Dashed horizontal line indicates the threshold (value at half-max) of the neural encoding nonlinearity. C: Filtered strain passed through the nonlinear function gives probability of spiking over time, $\mathrm{P}$ (fire). D: Spiking responses for 10 wingbeats each of the no rotation and rotation conditions. Spikes are generated probabilistically from $\mathrm{P}$ (fire), resulting in variable spike timing from wingbeat to wingbeat. Inset: Magnified view of the first firing event, surrounded by gray box, between 7.5 and 8.5 ms. E: Histogram of spike times (PSTHs) for each condition, summarizing spike timing over hundreds of wingbeats.

https://doi.org/10.1371/journal.pcbi.1009195.g002

interact with wing stiffness by simultaneously varying both wing stiffness and the threshold of the nonlinearity in neural encoding.

\section{Structure and neural encoding interact to determine accuracy and optimal sensor locations}

The neural threshold determines how selective the sensor will be for the feature given by the linear filter, with higher thresholds imparting stronger selectivity. The slope of the nonlinearity most strongly affects spike timing precision, with higher slopes leading to faster transitions from $\mathrm{P}($ fire $)=0$ to 1 and therefore greater spike timing precision. We set the slope of the nonlinearity to roughly match the spike timing precision observed in wing mechanosensors (0.1-1 $\mathrm{ms}$, [13]). The frequency content of the linear filter is chosen based on experimental observations [14]. Higher-frequency filters show uniformly low performance as a function of wing stiffness, while lower frequency filters show uniformly high performance (Fig A in S1 Appendix).

The ability to detect body rotations depends jointly on wing stiffness and neural threshold, with very dissimilar combinations yielding similarly high classification accuracy (Fig 3A). For 
A

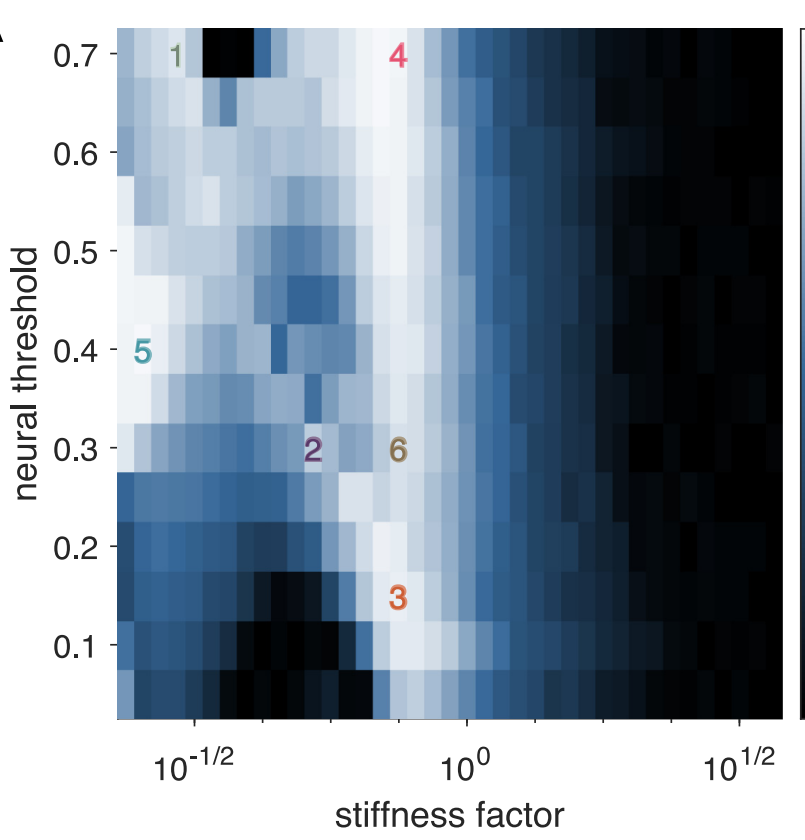

C

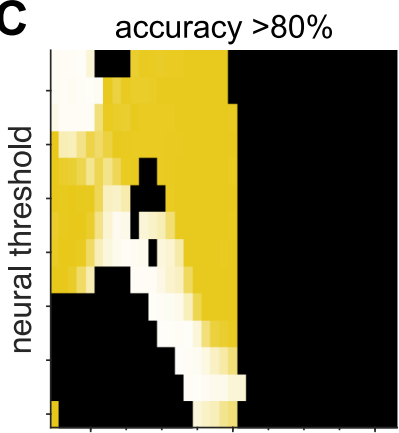

stiffness factor

stiffness factor
$100 \%$

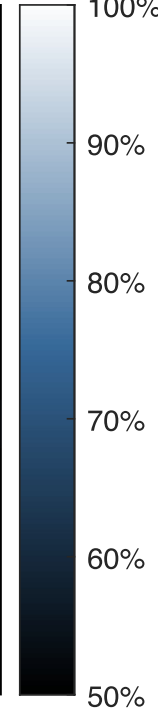

B

yaw
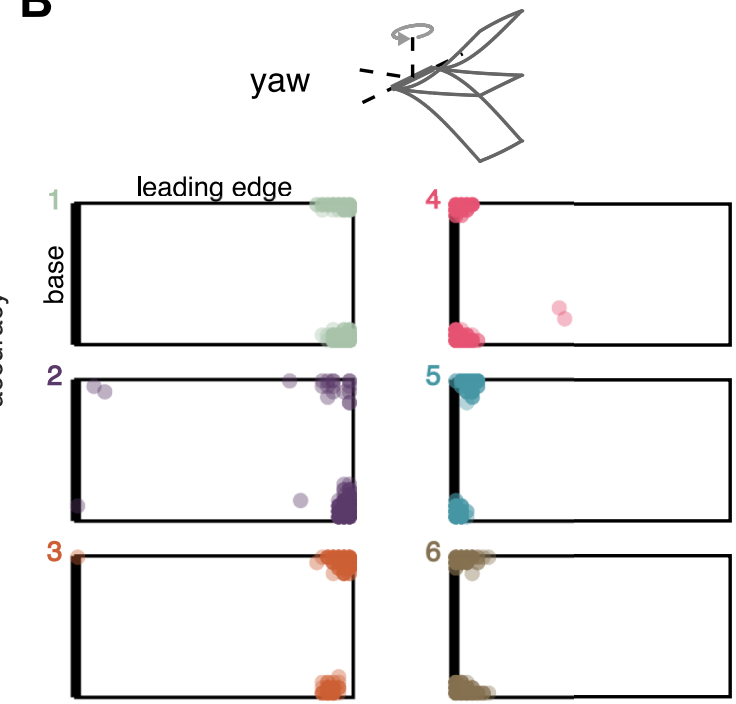

Fig 3. Wing stiffness and neural threshold interact to determine sensor locations and classification accuracy in the yaw axis. A: Accuracy as a function of neural threshold and wing stiffness. B: Optimal sensor locations of 10 best sensors, overlaid for 20 different simulated data sets. Neural threshold and wing stiffness of each panel are indicated by the corresponding colored dot in A. Top: Schematic indicates the axis of rotation. C: Optimal sensor locations in the spanwise direction from wing base (yellow) to wing tip (white) as a function of neural threshold and wing stiffness. Each panel shows results for a different accuracy cutoff. Black indicates parameter combinations that fall below the cutoff.

https://doi.org/10.1371/journal.pcbi.1009195.g003

example, for stiffness factors just below 1, accuracy is similarly high for low (0.15) and high

(0.7) thresholds, but accuracy is lower at intermediate values (e.g., 0.3).

Moreover, the optimal sensing strategies for a given wing stiffness can be qualitatively different for high and low thresholds. At stiffness factors just below 1, optimal sensors are located at the corners of the wing tip for low thresholds (Fig 3B, orange) and at the corners of the wing base for high thresholds (Fig 3B, pink). Note that sensors are expected to fall at the wing corners, as observed in previous work [23], because rotation elicits a twisting mode in wing bending, which leads to the largest differences in strain at wing corners between rotating and nonrotating conditions [31]. However, the distinction between sensors located at the wing base and those at the wing tip was not seen in previous work and is not necessarily expected [23]. When the spanwise sensor location (wing base to wing tip) is plotted as a function of neural threshold and wing stiffness, distinct regions of qualitatively different encoding strategies 
emerge (Fig 3C). Interestingly, these regions have rather convoluted, discontinuous shapes, illustrating that the optimal sensing strategy reflects the complex interaction of structural properties and neural encoding properties. Sensing accuracy and sensor locations are minimally impacted if sensors are allowed to be located on two identical wings, one on each side of the body (Fig B in S1 Appendix).

\section{For different classification tasks, a simple encoding principle emerges}

We next perform a similar set of numerical experiments for detecting rotation in each of the roll and pitch axes. Classification accuracy is generally lower for pitch detection compared to roll and yaw, with maximum accuracy across all parameter combinations tested only 77\% (Fig 4). Several clear differences emerge between classification of roll and pitch compared to yaw. First, for roll and pitch, classification accuracy generally increases with increasing wing stiffness, whereas accuracy for yaw generally decreases with increasing wing stiffness. Second, optimal sensors are distributed along the wing base for roll and pitch for nearly all parameters tested (Fig 4B and 4D). This observation is consistent with the fact that twisting modes elicited by pitch and roll are much smaller or nonexistent, respectively, compared to yaw. For pitch and roll, there is a far more straightforward relationship between wing stiffness and accuracy, with little dependence on neural threshold. A single encoding strategy emerges, with optimal sensors at the wing base.

Thus far, we have considered rotation about three orthogonal axes individually. However, an animal potentially needs to detect rotation about multiple axes, or rotation about axes intermediate to primary body axes. We assessed optimal sensing strategies in each of these cases and found that they reflect a combination of the strategies used for single-axis detection, rather than fundamentally different strategies (Figs D, E, and F in S1 Appendix).

\section{Robustness to perturbations}

To determine the extent to which these results are robust to different perturbations, we test two types of perturbations that reflect likely disruptions to both biological and engineered systems: sensor loss and external disturbances. Sensor loss may arise from wing damage sustained over an animal's lifetime or due to sensor failure in engineered systems. We select two wing stiffness/neural threshold parameter combinations that yield near-peak accuracy ( $\sim 100 \%$ for yaw and roll rotation; $\sim 75 \%$ for pitch rotation). For yaw axis rotation, one parameter set results in sensors placed at the wing base and one at the wing tip; for pitch and roll, both are located at the wing base. We randomly eliminate between 1 and 9 out of 10 sensors and compute accuracy using the remaining sensors. Accuracy falls smoothly as sensors are randomly dropped with, surprisingly, a single sensor still providing $~ 75 \%$ accuracy on average for yaw and roll, and $\sim 60 \%$ accuracy for pitch (Fig 5A, 5B and 5C). Accuracy falls more gradually for pitch detection, likely due to the lower starting accuracy.

We next examine the effects of external disturbances to wing rotation, which may arise from environmental fluctuations such as wind gusts. For yaw, pitch, and roll detection, accuracy falls smoothly as a function of the disturbance magnitude (Fig 5D, 5E and 5F). The details of how accuracy falls depend on the specific pairing of nonlinear threshold and wing stiffness: while accuracy typically decreases gradually with increasing disturbance, one case (low threshold for roll detection) exhibits a sharper drop in performance. Nevertheless, in all cases, accuracy remains well above chance for disturbances that are large relative to the magnitude of rotation, even when the standard deviation of the disturbance is the same magnitude as the rotation to be detected. 
A

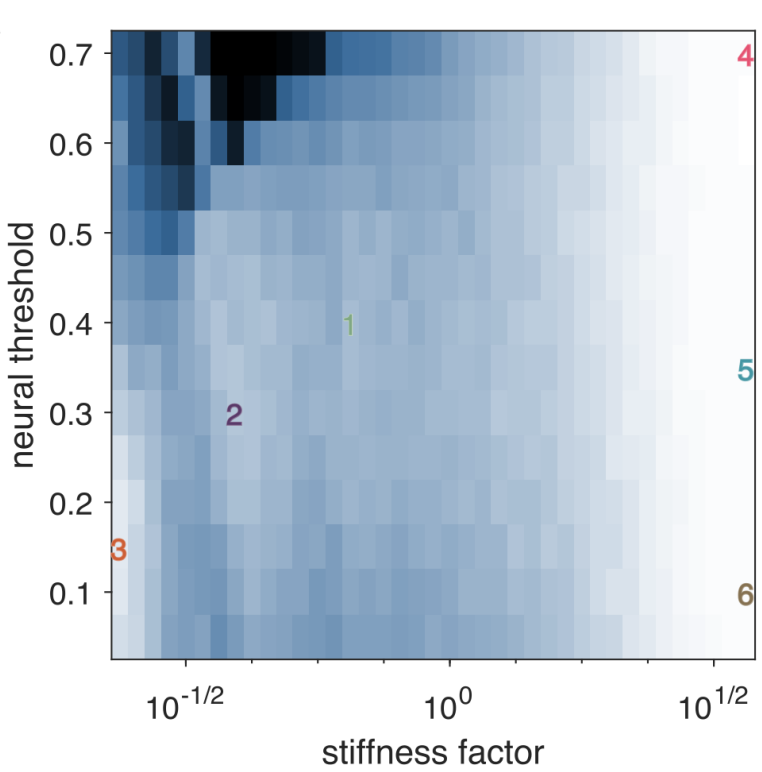

C

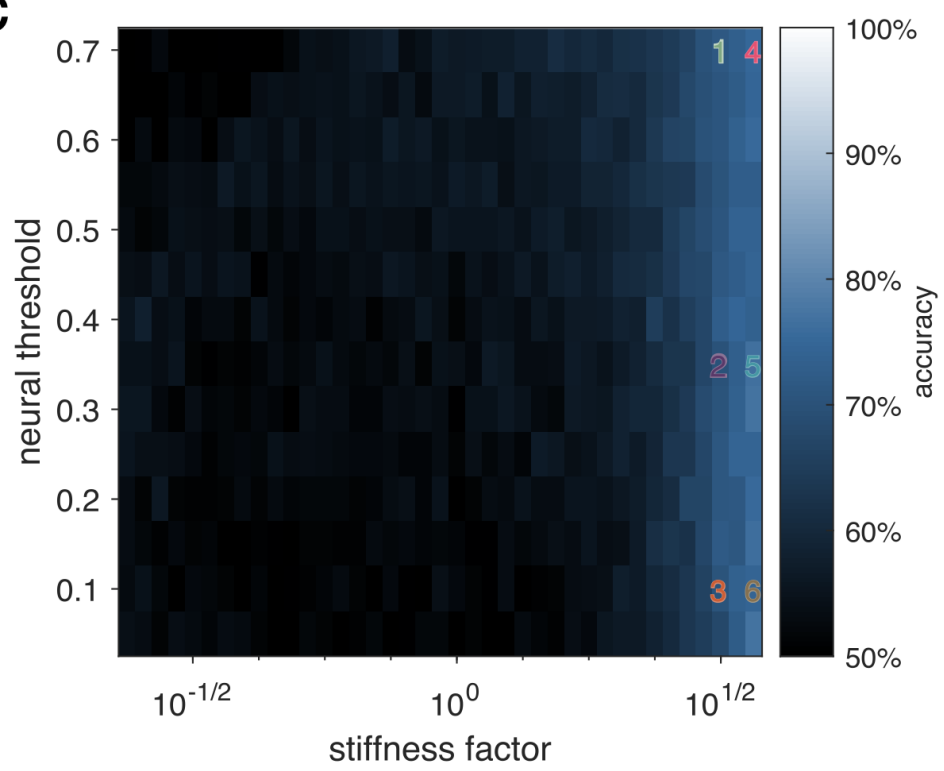

B

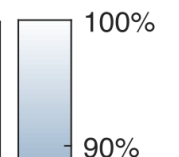

roll

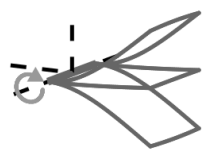

5

$80 \%$

leading edge
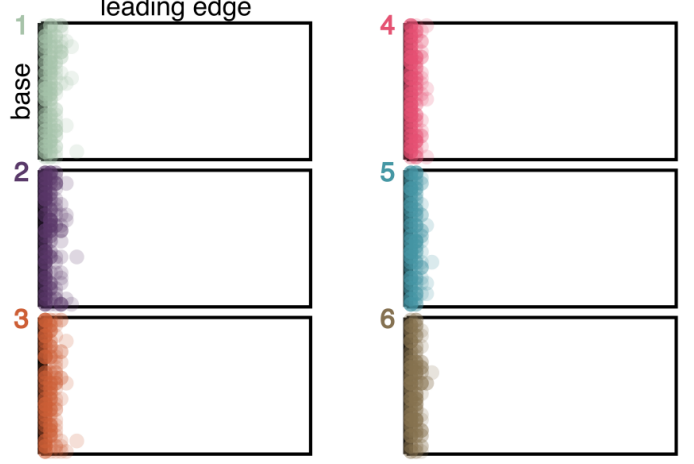

D
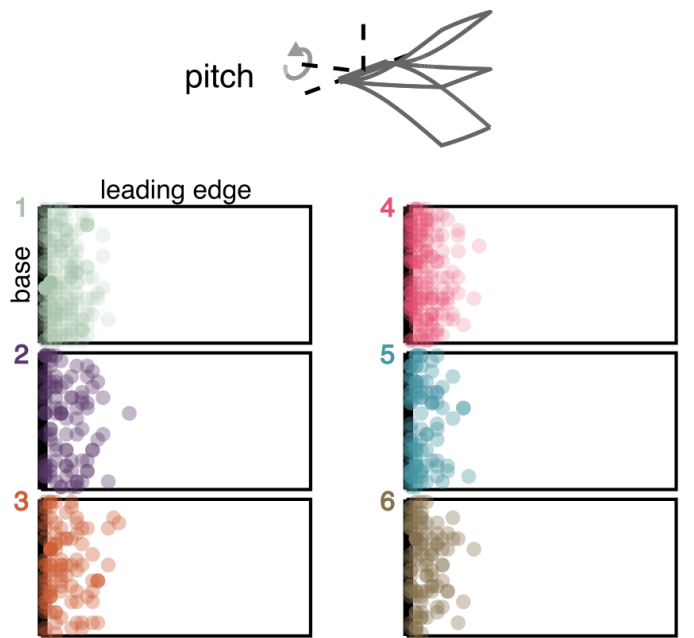

Fig 4. For detecting rotation in the roll and pitch axes, sensor locations do not depend on stiffness or neural encoding properties. A,C: Accuracy as a function of neural threshold and wing stiffness for roll and pitch, respectively. B,D: Optimal sensor locations of 10 best sensors, overlaid for 20 different data sets. Neural threshold and wing stiffness of each panel are indicated by the corresponding colored number in A. Top: Schematics indicate the axis of rotation.

https://doi.org/10.1371/journal.pcbi.1009195.g004

In summary, accuracy falls smoothly as the strength of a perturbation increases, with no indication of catastrophic failures at some threshold level of perturbation. Moreover, the trends are nearly identical for very different sensors, even when those sensors are located on opposite ends of the wing and often when they have very different neural thresholds. We therefore see no evidence of systematic differences in robustness between sensors at different locations or with different thresholds. 
A

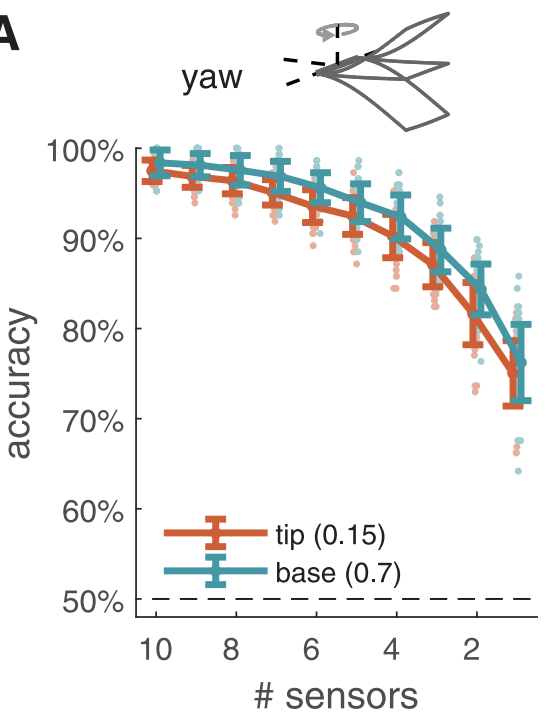

D

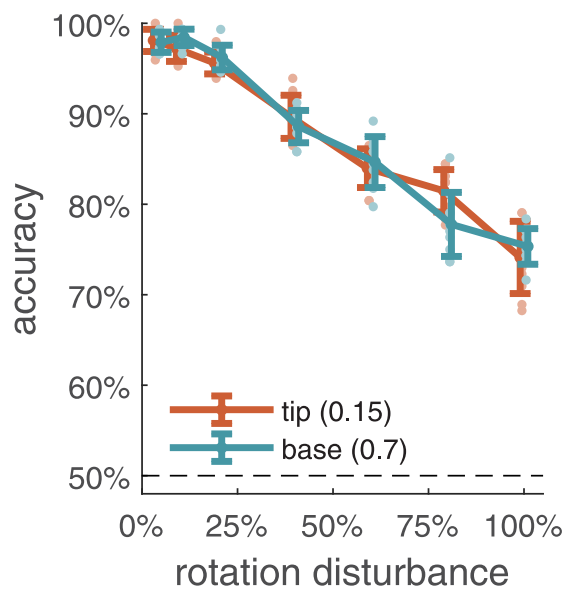

B
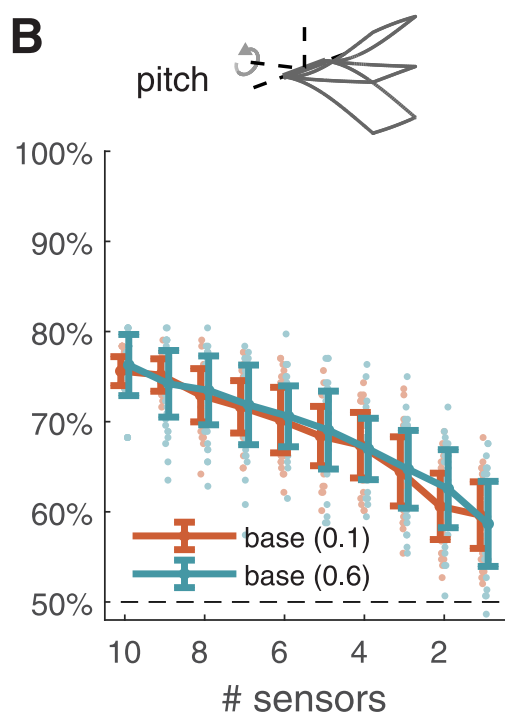

E

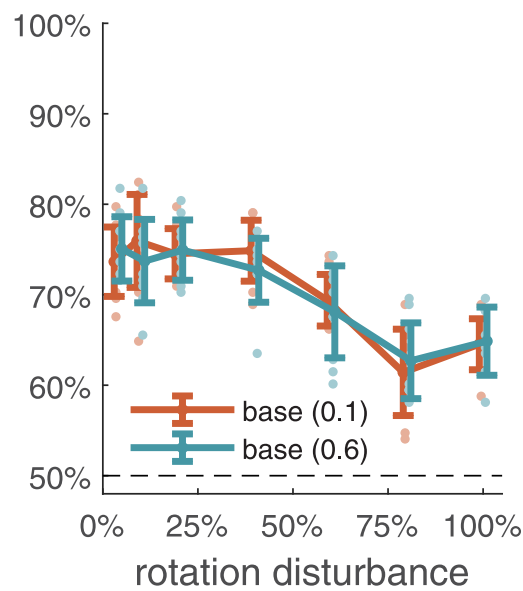

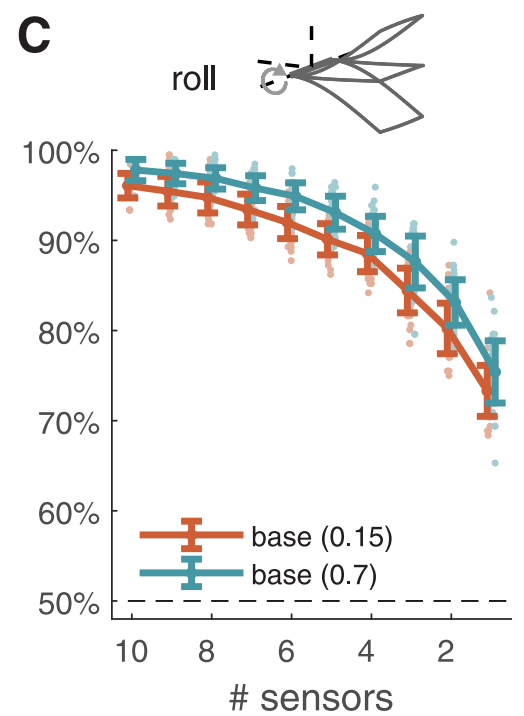

$\mathbf{F}$

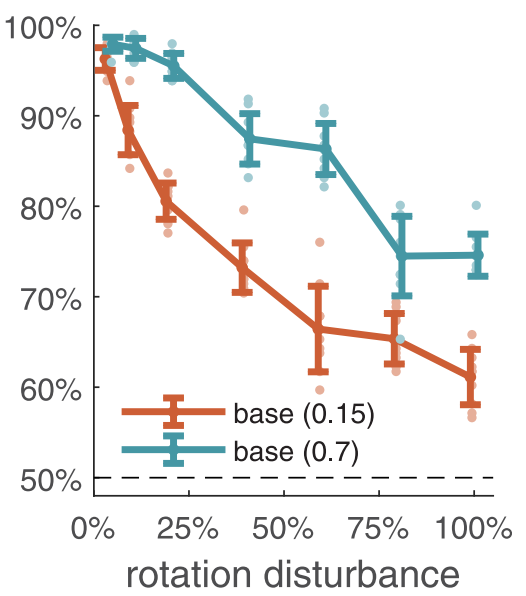

Fig 5. Robustness to sensor dropout and external disturbance. A: Accuracy of detecting rotation in the yaw axis as a function of number of sensors for two different wing stiffness/neural threshold parameter combinations (orange and teal). One parameter combination results in sensors at the wing tip, and a second results in sensors at the wing base, both with accuracy of $98 \%$ for 10 sensors. B-C: Same as A, for pitch and roll, respectively. In each case, two different parameter combinations were again chosen that produce similar accuracy with differing neural thresholds. For pitch-axis and roll-axis rotation detection, all sensors are located at the wing base. D-F: Accuracy as a function of the standard deviation of rotation disturbance for yaw, pitch, and roll, respectively, shown as the percentage of the constant rotation velocity to be detected. Parameter combinations same as corresponding A-C panels. Error bars in all panels denote \pm 1 standard deviation.

https://doi.org/10.1371/journal.pcbi.1009195.g005

\section{Discussion}

Our work is a first step towards understanding how insect wing structure determines incoming sensory information and thus sensing strategies. We examine the impacts of wing structure, in particular wing stiffness, on sensory encoding in a computational model of a flapping wing. We show that a small number of sensors, conveying spatially and temporally sparse signals can be used to reliably detect rotation over a range of wing stiffness values. Optimal encoding properties of these sensors and their placement vary with both wing stiffness and the need to identify rotation in different axes. Sensors are clustered at either the wing base or wing tip, depending on a combination of these factors. Moreover, accuracy is robust to multiple 
types of perturbations, and robustness does not depend on the particular details of sensor placement. The interaction of wing structure and neural encoding properties points to the importance of considering how these features may evolve together to enable sensing.

\section{Optimal sensing strategies for flight in engineered and biological systems}

Designing sensing strategies-including sensor number, placement, response properties, and sensor readout-has long been of interest to engineers who seek to build efficient, lightweight, low-cost systems. Much work in the domain of flight has focused on sensing in fixed-wing aircraft, rather than in flapping flight as exhibited by flying animals [32-36]. In a similar vein, these principles of optimality, efficient sensing, sparsity, and cost reduction have also informed neuroscientists' understanding of the nervous system [26, 37-40]. In many cases, the encoding properties of sensory neurons are matched to the statistics of the incoming signals. These encoding properties may be tuned over many timescales, from evolutionary time, to dynamically on subsecond timescales to match changing statistics in the environment [41, 42]. Therefore, understanding optimal sensing strategies provides a useful benchmark for understanding biological systems.

Prior studies have considered optimal strain sensing strategies in insect wings [23, 43-46]. Although using different sensing tasks and optimization criteria, taken together, they show that remarkably few sensors are needed to detect or identify patterns of strain on the wing. However, all of the previous work assumes sensors that either directly sense strain or encode strain as a continuous variable. In contrast, our current work uses spiking sensors. Spiking sensors are not only more faithful to the corresponding biological systems, where primary sensory neurons on the wing encode information in all-or-none action potentials, but they also enforce that sensors transmit temporally sparse signals. Investigating spiking sensors could present opportunities for more efficient, bio-inspired sensing approaches in engineered systems as well.

\section{Strain sensor placement in insect wings}

Insect wings exhibit widely varying patterns of mechanosensor placement on their wings [4751]. Even so, we have little understanding of the functional consequences of this diversity.

Moreover, comparison between species is complicated by the vast differences in wing morphology and stiffness $[29,52]$. Despite these differences, some generalizations can be made about the placement of strain sensors (campaniform sensilla). Perhaps the most striking commonality across diverse taxa is the relative abundance of campaniform sensilla near the wing base $[47-49,51,53-55]$. Taken together, the results in the present work suggest that clustering sensors at the wing base may serve as a catch-all strategy, favorable across a wide range of wing and neural encoding properties to detect rotations about multiple axes (Figs 3 and 4 and Fig F in S1 Appendix).

\section{Additional features driving optimal sensor placement}

Insect species vary widely in features that are likely to play a role in determining effective mechanosensor placement on the wings: wing size, shape, and venation pattern, among others. Understanding the functional consequences of these different features and how they impact spatiotemporal patterns of strain on the wing will be necessary for elucidating principles guiding the differing placement of sensory structures across diverse insect taxa. Answering these questions will require different computational models that allow more flexible manipulation of these variables. For example, models based on the finite element method (FEM) support more realistic wing geometry, wing venation patterns, and non-uniform stiffness [29, 49]. The 
consequences of many of these properties have been previously explored in a variety of models, although none from a sensing perspective $[18,19,29,56,57]$. More biologically realistic features will result in more complex spatiotemporal patterns of wing strain, likely driving sensor placement to be far more complex and varied than those uncovered in the current study.

The wingstroke trajectory and kinematics may play a particularly important role in determining optimal sensor placement. In the current work, we see that the wingstroke, modeled as a sum of sines as in previous work [23,31], drives two distinct response events over the course of single wingbeat (Fig 2). Interestingly, these response events interact with spike timing precision in sometimes counterintuitive ways, such that lower spike timing precision can sometimes yield greater discriminability. More complex wingstroke kinematics likely play an important role in determining the phase (or phases) within a wingstroke when each neuron is most sensitive. The trajectory of the wingstroke may also be used as a mechanism for active sensing, in which the animal modifies its motor output to acquire more useful sensory information [58-62]. Understanding the consequences of varying motor output for incoming sensory information will be crucial to understanding sensing strategies in flight.

Although we varied neural encoding properties (nonlinear threshold and filter frequency; Fig 3 and Fig A in S1 Appendix), all sensors on a given wing have identical encoding properties. Previous work suggests that there are likely two sub-populations of strain-sensitive neurons on the wings of moths [63]. It is possible that a wing with different sensor types may need fewer sensors and adopt a qualitatively different encoding strategy. Additionally, selectivity to multiple features has been demonstrated in neurons in multiple sensory systems, including strain-sensitive neurons in the halteres of crane flies [6,64]. Allowing individual sensors to respond to multiple stimulus features may also allow for fewer sensors and different sensor positioning. However, it has recently been suggested that all strain-sensitive neurons on insect wings may, in fact, respond similarly in the context of flight (i.e., for rapidly fluctuating stimuli), suggesting that sensor placement may be the primary determinant of information encoded by a given sensor [65].

The current work investigates sensing strategies by finding solutions to a set of optimization tasks. However, biological systems need not operate at any true optimum, but may instead simply need to be "good enough" or robust to failure [66]. Solving for optimal sensor locations likely explains some of the features of the present study, for example, the abrupt shift between wing base and wing tip sensing strategies for yaw detection (Fig 3). If sensor locations are restricted to either the distal or the proximal half of the wing, sensor performance does not fall off sharply at these same transitions (Fig G in S1 Appendix). We investigated robustness of the optimal sensing strategies in the current study and found little difference between different sensor properties (locations or threshold), suggesting that a need for robustness would not favor one of these strategies over another. Exploring the range of sensing strategies that meet some (sub-optimal) performance criterion may be an interesting area of future work.

\section{Sensing needs are one of many evolutionary demands on wing structure}

An organism's evolution reflects a combination of evolutionary constraints and an organism's needs $[67,68]$. For insect wings, these needs may include force generation for flight, sensing, mating display, and predator avoidance, among others [69-71]. How wing structure impacts aerodynamic performance has been extensively studied [17-20, 22, 72-74]. These studies generally point to the importance of stiffness gradients (from base to tip and from leading edge to trailing edge) and rigidity provided by wing veins. However, the consequences of wing structure for sensing have remained unexplored, despite the fact that insect wings have long been known to provide sensory feedback during flight $[12,75,76]$. 
Incorporating additional constraints may shed light on how these needs interact. For example, in insects, campaniform sensilla on the wings are generally restricted to be on or near wing veins $[47,53,63,77]$. Because we used a highly simplified wing model that did not include wing veins (or any spatial variation in stiffness), we allowed sensors to be placed at any point on a dense grid over the surface of the wing. Constraining sensor locations is straightforward and can be achieved by incorporating an additional penalty in the optimization step to decrease the likelihood of sensors being located in particular regions.

In the present work, we focus on how wing structure affects sensing and do not explore how sensing might interact with other roles of the wing, such as actuation, predator avoidance, and mating display. An important, and challenging, open question is: how are these diverse needs balanced over evolutionary timescales? Answering this question demands an interdisciplinary approach that combines biomechanics, neuroscience, and evolutionary biology to produce a more complete understanding of the processes driving wing evolution.

\section{Methods}

Code that implements strain simulations, conversion of strain to spiking data, and sensor optimization can be found at https://github.com/aiweber/optimal_sensing_ELwing.

\section{Euler-Lagrange simulations of wing strain}

We first simulate spatiotemporal patterns of strain over the surface of the wing using a previously developed Euler-Lagrange model based on parameters of wings of the hawkmoth Manduca sexta [31]. In this model, the wing is represented by a flat plate with a span of $50 \mathrm{~mm}$, chord length of $25 \mathrm{~mm}$, and thickness of $0.127 \mathrm{~mm}$. Following prior modeling efforts [31], wing flapping motion is modeled as a sum of sines, with a primary frequency of $25 \mathrm{~Hz}$ and a secondary frequency of $50 \mathrm{~Hz}$ ( $76 \%$ amplitude). The wing is subject to rotation in different axes of comparable magnitude to rotations experienced by a hawkmoth during free flight (constant at $10 \mathrm{rad} / \mathrm{s}$ unless otherwise noted) [78]. Additionally, noise is added to both the flapping velocity as well as the rotation rate to simulate noise experienced during flapping flight, as in previous work [23]. Unless otherwise noted, the magnitude of noise in rotation is $1 \%$ of the rotation rate, and the magnitude of noise in flapping is $2 \%$ of the flapping amplitude. For the roll detection task, $2 \%$ noise in flapping is included with no additional noise in rotation, as rotation occurs in the same axis as flapping. For the four-way classification task, noise in all axes is included in all conditions.

We vary Young's modulus to explore the effects of changing structural properties of the wing, namely wing flexural stiffness. The range of explored modulus values is approximately centered at $3 \mathrm{GPa}$ (stiffness factor $=1$ ), corresponding to a spanwise flexural stiffness of $\sim 1.5^{*}$ $10^{-4} \mathrm{Nm}^{2}$, comparable to average experimentally measured spanwise flexural wing stiffness in Manduca sexta [28]. We vary Young's modulus values from $0.7 \mathrm{GPa}$ to $10.0 \mathrm{GPa}$ (flexural stiffness $3.6^{*} 10^{-5}$ to $5.6^{*} 10^{-4} \mathrm{Nm}^{2}$ ). This approximately corresponds to the flexural stiffness range from half the wing span to the wing base [29]. Below 0.7 GPa, simulations did not achieve appropriate levels of numerical convergence, and more flexible wings therefore cannot be tested with this model.

We simulate strain over a dense grid representing sensor locations with $1 \mathrm{~mm}$ spacing, resulting in 26 chordwise and 51 spanwise sensors, for a total of 1,326 sensor locations. Unless otherwise noted, we use normal strain in the spanwise direction. This provided improved performance over normal strain in the chordwise direction (Fig H in S1 Appendix). See [31] for additional details of Euler-Lagrange simulations and [23] for additional details of simulations 
with disturbances. We typically simulate 3 seconds of data ( 75 wingbeats) for each condition at a sampling rate of $10 \mathrm{kHz}$.

\section{Strain encoding with neural-inspired spiking sensors}

Neural-inspired sensors encode strain following a procedure similar to earlier work [23]. At each sensor location, strain is first convolved with a linear filter, representing the temporal feature of strain which the sensor is most sensitive to. The filtered strain signal reflects the similarity between linear filter (i.e., feature) and the strain experienced by the sensor over time.

Filtered strain is then transformed by a static nonlinearity, which determines the sensor's selectivity for that particular feature: sensors with high threshold will only respond when the temporal pattern of strain is very similar to the feature given by the linear filter, corresponding to strong selectivity. The output of the linear-nonlinear encoding represents a probability that the neuron will generate a spike.

The shapes of the linear filter and nonlinearity are based on previous electrophysiological recordings of responses in mechanosensors of the wing nerve [14]. The linear filter $f$ is defined as a decaying sinusoidal function:

$$
f(t, \omega, \tau, \delta)=\cos (2 \pi \omega(t+\tau)) \cdot \exp \left(\frac{-(t+\tau)^{2}}{\delta^{2}}\right)
$$

where $\omega$ is the frequency of the filter, $\tau$ is the time offset to the peak, and $\delta$ is the decay time. In this work, $\omega=\frac{1}{2 \pi} \mathrm{ms}^{-1}, \tau=5 \mathrm{~ms}$, and $\delta=4 \mathrm{~ms}$. The static nonlinearity (also called the nonlinear decision function or nonlinear activation function) is given by:

$$
N\left(g_{t}, \alpha, \beta\right)=\frac{1}{1+\exp \left(-\alpha\left(g_{t}-\beta\right)\right)}
$$

where $g_{t}$ is the filtered stimulus at time $t, \alpha$ is the slope parameter, and $\beta$ is the threshold parameter, where the function reaches half-maximum. We hold $\alpha$ constant at 50 and vary the threshold $\beta$. As in previous work, $g$ is normalized by a constant (identical for all sensors) such that the maximum value of $g$ over all sensors is approximately 1 for wing stiffness of $3 \mathrm{GPa}$. Threshold is varied between 0.05 and 0.7 . (The normalization constant is only important insofar as it sets the scale of the threshold parameter. The range of thresholds we test therefore represent about $5-70 \%$ of the maximum filtered stimulus.)

We then generate spikes probabilistically from the output of the linear-nonlinear encoding. The sensor spikes if the probability of firing exceeds a random draw from a standard uniform distribution. We manually impose an absolute refractory period of $15 \mathrm{~ms}$ between spikes. This is not intended to represent the actual absolute refractory period of mechanosensors, but rather to empirically match observations from previous experimental work that each sensor fires only 1-2 spikes per wingbeat $[75,79]$. For each data set simulated from the EulerLagrange model, we generate 10 sets of spiking responses, for a total of 1,500 data points per optimization ( 75 wingbeats per Euler-Lagrange simulation, 10 sets of spikes generated for each simulation, for both flapping only and flapping with rotation). Noise in the spike generation step dominates noise in the Euler-Lagrange simulations, so similar results would be expected for shorter Euler-Lagrange simulations and more repetitions of spiking responses generated for each simulation. 


\section{Sensor optimization}

Our objective in sensor optimization is to determine the placement of a small number of neural-inspired sensors which can be used to determine whether or not the animal is rotating. We simulate spiking data as described above for two cases: one where a wing is flapping, and one where a wing is flapping and rotating (in either the yaw or pitch axis). For both of these cases, we determine the time to first spike within each wingbeat with $0.1 \mathrm{~ms}$ precision and use only this information to classify the data. For wingbeats where no spike is elicited, we designate the spike time as zero, though results are unchanged if we instead designate the spike time as a time longer than the wingbeat duration (e.g., $400 \mathrm{~ms}$ spike time compared to $40 \mathrm{~ms}$ period). Data are standardized in this optimization step, but the original (non-standardized) data are used to evaluate accuracy.

To determine optimal sensor locations, we use a previously developed method called sparse sensor placement optimization for classification (SSPOC) [80]. This method first uses dimensionality reduction (principal component analysis, in our case) to find a lower-dimensional subspace that captures important features of the data. We then use a linear discriminant analysis (LDA) to find the projection vector $w$ that maximally separates the classes of our data in this subspace. Finally, we use elastic net regularization to solve for a sparse set of sensors $s$ that can reconstruct the projection vector $w$. For two-way classification (flapping only, rotation about a single axis), we solve:

$$
s=\underset{s^{\prime}}{\arg \min }\left[\lambda\left\|s^{\prime}\right\|_{1}+(1-\lambda)\left\|s^{\prime}\right\|_{2}\right] \quad \text { subject to } \Psi^{T} s^{\prime}=w,
$$

where $s$ is a vector of sensor weights ( $n \times 1$, with many near-zero entries), $\Psi$ is the low-dimensional basis $(n \times m, m<n), w$ is the projection vector in the low-dimensional subspace ( $m \times 1)$, and $\lambda$ determines the balance between $L_{1}$ and $L_{2}$ regularization. We set $m=3$ and $\lambda=0.9$.

We also perform a four-way classification of: (1) flapping only, (2) rotation in yaw, (3) rotation in pitch, and (4) rotation in roll. In this case, we perform the same elastic net regularization, subject to a different constraint:

$$
s=\underset{s^{\prime}}{\arg \min }\left[\lambda\left\|s^{\prime}\right\|_{1}+(1-\lambda)\left\|s^{\prime}\right\|_{2}\right] \quad \text { subject to } \Psi^{T} s^{\prime}-w \leq \epsilon,
$$

where $\epsilon=10^{-6}$.

We use the cvx package to solve this optimization problem (http://cvxr.com/cvx/) [81, 82].

\section{Performance evaluation}

$90 \%$ of the data is used as a training set for the optimization, and $10 \%$ is held out as test data to evaluate accuracy. For straightforward comparison across conditions, we consistently use the top 10 sensors (i.e., sensors with the largest weights in $s$ ) to assess classification accuracy. We find that 10 sensors are typically enough to achieve near-peak accuracy (Fig 5A, 5B and 5C) without including a large number of extraneous sensors. LDA is again used to find the best projection vector $w_{c}$ for the non-standardized test data for only the top 10 sensors. For twoway classification, a decision boundary is drawn at the mean of the two condition centroids. For four-way classification, we classify individual points according to the nearest centroid. (Note that the results will be the same whether or not the test data set is standardized. We choose to calculate accuracy based on the non-standardized data to highlight the fact that a linear decoding scheme can be used to read out information from the original spike-timing data.) 


\section{Supporting information}

S1 Appendix. Further examination of factors affecting optimal sensor locations and sensing performance.

(PDF)

\section{Acknowledgments}

We would like to thank Thomas Mohren and Michelle Hickner for helpful discussions that shaped this project and for contributions to code development.

\section{Author Contributions}

Conceptualization: Alison I. Weber, Thomas L. Daniel, Bingni W. Brunton.

Investigation: Alison I. Weber, Thomas L. Daniel, Bingni W. Brunton.

Methodology: Alison I. Weber, Thomas L. Daniel, Bingni W. Brunton.

Software: Alison I. Weber.

Supervision: Thomas L. Daniel, Bingni W. Brunton.

Writing - original draft: Alison I. Weber.

Writing - review \& editing: Alison I. Weber, Thomas L. Daniel, Bingni W. Brunton.

\section{References}

1. Dallos P. The Active Cochlea. Journal of Neuroscience. 1992; 12(12):4575-4585. https://doi.org/10. 1523/JNEUROSCI.12-12-04575.1992 PMID: 1464757

2. Loewenstein WR, Mendelson M. Components of Receptor Adaptation in a Pacinian Corpuscle. Journal of Physiology. 1965; 177:377-397. https://doi.org/10.1113/jphysiol.1965.sp007598

3. Barth FG. Mechanics to pre-process information for the fine tuning of mechanoreceptors. Journal of Comparative Physiology A: Neuroethology, Sensory, Neural, and Behavioral Physiology. 2019; 205 (5):661-686. https://doi.org/10.1007/s00359-019-01355-z PMID: 31270587

4. Birdwell JA, Solomon JH, Thajchayapong M, Taylor MA, Cheely M, Towal RB, et al. Biomechanical models for radial distance determination by the rat vibrissal system. Journal of Neurophysiology. 2007; 98(4):2439-2455. https://doi.org/10.1152/jn.00707.2006 PMID: 17553946

5. Sen K, Theunissen FE, Doupe AJ. Feature analysis of natural sounds in the songbird auditory forebrain Journal of Neurophysiology. 2001; 86(3):1445-1458. https://doi.org/10.1152/jn.2001.86.3.1445 PMID: 11535690

6. Fairhall AL, Burlingame C, Narasimhan R, Harris R, Puchalla J, Berry MJ. Selectivity for multiple stimulus features in retinal ganglion cells. Journal of Neurophysiology. 2006; 96(5):2724-38. https://doi.org/ 10.1152/jn.00995.2005 PMID: 16914609

7. Bale MR, Maravall M. Organization of sensory feature selectivity in the whisker system. Neuroscience. 2018; 368:70-80. https://doi.org/10.1016/j.neuroscience.2017.09.014 PMID: 28918260

8. Chichilnisky E. A simple white noise analysis of neuronal light responses. Network. 2001; 12:199-213. https://doi.org/10.1080/713663221 PMID: 11405422

9. Slee SJ, Higgs MH, Fairhall AL, Spain WJ. Two-dimensional time coding in the auditory brainstem. Journal of Neuroscience. 2005; 25(43):9978-88. https://doi.org/10.1523/JNEUROSCl.2666-05.2005 PMID: 16251446

10. Wark B, Lundstrom BN, Fairhall AL. Sensory adaptation. Current Opinion in Neurobiology. 2007; 17 (4):423-9. https://doi.org/10.1016/j.conb.2007.07.001 PMID: 17714934

11. Aiello BR, Westneat MW, Hale ME. Mechanosensation is evolutionarily tuned to locomotor mechanics. Proceedings of the National Academy of Sciences. 2017; 114(17):4459-4464. https://doi.org/10.1073/ pnas.1616839114 PMID: 28396411 
12. Elson RC. Integration of wing proprioceptive and descending exteroceptive sensory inputs by thoracic interneurones of the locust. Journal of Experimental Biology. 1987; 128:193-217. https://doi.org/10. 1242/jeb.128.1.193

13. Dickerson $\mathrm{BH}$, Aldworth ZN, Daniel TL. Control of moth flight posture is mediated by wing mechanosensory feedback. Journal of Experimental Biology. 2014; 217(13):2301-2308. PMID: 24737754

14. Pratt $B$, Deora $T$, Mohren $T$, Daniel T. Neural evidence supports a dual sensory-motor role for insect wings. Proceedings of the Royal Society B: Biological Sciences. 2017; 284 (1862). https://doi.org/10. 1098/rspb.2017.0969 PMID: 28904136

15. Bomphrey RJ, Godoy-Diana R. Insect and insect-inspired aerodynamics: unsteadiness, structural mechanics and flight control. Current Opinion in Insect Science. 2018; 30:26-32. https://doi.org/10. 1016/j.cois.2018.08.003 PMID: 30410869

16. Taylor GK, Krapp HG. Sensory systems and flight stability: what do insects measure and why? Advances in Insect Physiology. 2007; 34:231-316. https://doi.org/10.1016/S0065-2806(07)34005-8

17. Bomphrey RJ, Nakata T, Henningsson $P$, Lin HT. Flight of the dragonflies and damselflies. Philosophical Transactions of the Royal Society B: Biological Sciences. 2016; 371(1704):20150389. https://doi. org/10.1098/rstb.2015.0389 PMID: 27528779

18. Eberle AL, Reinhall PG, Daniel TL. Fluid-structure interaction in compliant insect wings. Bioinspiration and Biomimetics. 2014; 9(2). https://doi.org/10.1088/1748-3182/9/2/025005 PMID: 24855064

19. Mountcastle AM, Daniel TL. Vortexlet models of flapping flexible wings show tuning for force production and control. Bioinspiration and Biomimetics. 2010; 5(4). https://doi.org/10.1088/1748-3182/5/4/045005 PMID: 21098955

20. Nakata T, Liu H. A fluid-structure interaction model of insect flight with flexible wings. Journal of Computational Physics. 2012; 231(4):1822-1847.

21. Zhao L, Huang Q, Deng X, Sane SP. Aerodynamic effects of flexibility in flapping wings. Journal of the Royal Society Interface. 2010; 7(44):485-497. https://doi.org/10.1098/rsif.2009.0200 PMID: 19692394

22. Song A, Tian X, Israeli E, Galvao R, Bishop K, Swartz S. Aeromechanics of Membrane Wings with Implications for Animal Flight. AIAA Journal. 2008; 46(8). https://doi.org/10.2514/1.36694

23. Mohren TL, Daniel TL, Brunton SL, Brunton BW. Neural-inspired sensors enable sparse, efficient classification of spatiotemporal data. Proceedings of the National Academy of Sciences. 2018; 115 (42):10564-10569. https://doi.org/10.1073/pnas.1808909115 PMID: 30213850

24. Giannakis GB, Mateos G, Farahmand S, Kekatos V, Zhu H. USPACOR: Universal sparsity-controlling outlier rejection. In: 2011 IEEE International Conference on Acoustics, Speech and Signal Processing (ICASSP). IEEE; 2011. p. 1952-1955.

25. Ahmad S, Scheinkman L. How can we be so dense? The benefits of using highly sparse representations. arXiv. 2019;1903.11257.

26. Niven JE, Laughlin SB. Energy limitation as a selective pressure on the evolution of sensory systems. Journal of Experimental Biology. 2008; 211:1792-1804. https://doi.org/10.1242/jeb.017574 PMID: 18490395

27. Srivastava N, Hinton GE, Krizhevsky A, Sutskever I, Salakhutdinov RR. Dropout: A Simple Way to Prevent Neural Networks from Overfitting. Journal of Machine Learning Research. 2014; 15:1929-1958.

28. Combes SA, Daniel TL. Flexural stiffness in insect wings I. Scaling and the influence of wing venation. Journal of Experimental Biology. 2003; 206(17):2979-2987. https://doi.org/10.1242/jeb.00523 PMID: 12878666

29. Combes SA, Daniel TL. Flexural stiffness in insect wings. II. Spatial distribution and dynamic wing bending. Journal of Experimental Biology. 2003; 206(17):2989-2997. https://doi.org/10.1242/jeb.00524 PMID: 12878667

30. Pass G. Beyond aerodynamics: The critical roles of the circulatory and tracheal systems in maintaining insect wing functionality. Arthropod Structure and Development. 2018; 47(4):391-407. https://doi.org/ 10.1016/j.asd.2018.05.004 PMID: 29859244

31. Eberle AL, Dickerson BH, Reinhall PG, Daniel TL. A new twist on gyroscopic sensing: Body rotations lead to torsion in flapping, flexing insect wings. Journal of the Royal Society Interface. 2015; 12(104). https://doi.org/10.1098/rsif.2014.1088 PMID: 25631565

32. Gherlone $M$, Cerracchio $P$, Mattone $M$. Shape sensing methods: Review and experimental comparison on a wing-shaped plate. Progress in Aerospace Sciences. 2018; 99:14-26. https://doi.org/10.1016/j. paerosci.2018.04.001

33. Mohamed A, Watkins S, Fisher A, Marino M, Massey K, Clothier R. Bioinspired Wing-Surface Pressure Sensing for Attitude Control of Micro Air Vehicles. Journal of Aircraft. 2015; 52(3):827-838. https://doi. org/10.2514/1.C032805 
34. Kefal A, Yildiz M. Modeling of Sensor Placement Strategy for Shape Sensing and Structural Health Monitoring of a Wing-Shaped Sandwich Panel Using Inverse Finite Element Method. Sensors. 2017; 17 (12):2775. https://doi.org/10.3390/s17122775 PMID: 29189758

35. Castano L, Airoldi S, McKenna T, Humbert J. Wing sensor placement for gust disturbance rejection. In: AIAA Atmospheric Flight Mechanics Conference; 2015. p. 0749.

36. Shin H, Castano LM, Humbert JS, Bergbreiter S. Sensing skin for detecting wing deformation with embedded soft strain sensors. In: 2016 IEEE SENSORS; 2016. p. 1-3.

37. Laughlin S. A Simple Coding Procedure Enhances a Neuron's Information Capacity. Zeitschrift f $\tilde{A} 1 / 4 r$ Naturforschung. 1981; 36:910-912. PMID: 7303823

38. Srinivasan MV, Laughlin SB, Dubs A. Predictive Coding: A Fresh View of Inhibition in the Retina. Proceedings of the Royal Society B: Biological Sciences. 1982; 216:427-459. PMID: 6129637

39. Olshausen BA, Field DJ. Emergence of simple-cell receptive field properties by learning a sparse code for natural images. Nature. 1996; 381(6583):607-609. https://doi.org/10.1038/381607a0 PMID: 8637596

40. Atick J, Redlich A. Towards a Theory of Early Visual Processing. Neural Comput. 1990; 2(3):308-320. https://doi.org/10.1162/neco.1990.2.3.308

41. Fairhall AL, Lewen G, Bialek W, de Ruyter Van Steveninck R. Efficiency and ambiguity in an adaptive neural code. Nature. 2001; 412(6849):787-92. https://doi.org/10.1038/35090500 PMID: 11518957

42. Bialek W, Owen WG. Temporal filtering in retinal bipolar cells: Elements of an optimal computation? Biophysical Journal. 1990; 58:1227-1233. https://doi.org/10.1016/S0006-3495(90)82463-2 PMID: 2291942

43. Hinson BT, Morgansen KA. Gyroscopic sensing in the wings of the hawkmoth Manduca sexta: The role of sensor location and directional sensitivity. Bioinspiration and Biomimetics. 2015; 10(5). https://doi. org/10.1088/1748-3190/10/5/056013 PMID: 26440705

44. Manohar K, Brunton SL, Kutz JN. Environment identification in flight using sparse approximation of wing strain. Journal of Fluids and Structures. 2017; 70:162-180. https://doi.org/10.1016/j.jfluidstructs. 2017.01.008

45. Boyacioglu B, Morgansen KA. Bioinspired Observability Analysis Tools for Deterministic Systems with Memory in Flight Applications. In: AIAA Scitech 2021 Forum; 2021. p. 1679.

46. Johns $W$, Davis $L$, Jankauski $M$. Reconstructing full-field flapping wing dynamics from sparse measurements. Bioinspiration \& Biomimetics. 2021; 16(1):016005. https://doi.org/10.1088/1748-3190/abb0cb

47. Dombrowski UJ. Untersuchungen zur funktionellen Organisation des Flugsystems von Manduca sexta (L.) [PhD thesis]. Universitaet zu Koeln; 1991.

48. Pringle JWS. Insect flight. Cambridge: Cambridge University Press; 1957.

49. Fabian J, Siwanowicz I, Uhrhan M, Maeda M, Bomphrey R, Lin HT. Complete neuroanatomy and sensor maps of Odonata wings for fly-by-feel flight control. bioRxiv. 2021.

50. Dickinson MH, Palka J. Physiological properties, time of development, and central projection are correlated in the wing mechanoreceptors of Drosophila. Journal of Neuroscience. 1987; 7(12):4201-4208. https://doi.org/10.1523/JNEUROSCI.07-12-04201.1987 PMID: 3694271

51. Schmidt JM, Smith JJB. The Ultrastructure of the Wings and the External Sensory Morphology of the Thorax in Female Trichogramma-Minutum Riley (Hymenoptera, Chalcidoidea, Trichogrammatidae). Proceedings of the Royal Society Series B-Biological Sciences. 1985; 224(1236):287-+.

52. Salcedo MK, Hoffmann J, Donoughe S, Mahadevan L. Computational analysis of size, shape and structure of insect wings. Biology Open. 2019; 8(10):1-9. https://doi.org/10.1242/bio.040774 PMID: 31628142

53. Gettrup E. Sensory regulation of wing twisting in locusts. Journal of Experimental Biology. 1966; 44:116. https://doi.org/10.1242/jeb.44.1.1 PMID: 5922737

54. Cole ES, Palka J. The Pattern of Campaniform Sensilla on the Wing and Haltere of Drosophila-Melanogaster and Several of Its Homeotic Mutants. Journal of Embryology and Experimental Morphology. 1982; 71(Oct):41-61. PMID: 6818316

55. Frantsevich L, Gorb S, Radchenko V, Gladun D, Polilov A. Lehr's fields of campaniform sensilla in beetles (Coleoptera): Functional morphology. I. General part and allometry. Arthropod Structure \& Development. 2014; 43(6):523-535. https://doi.org/10.1016/j.asd.2014.09.001 PMID: 25240964

56. Jankauski M, Shen IY. Dynamic modeling of an insect wing subject to three-dimensional rotation. International Journal of Micro Air Vehicles. 2014; 6(4):231-251. https://doi.org/10.1260/1756-8293.6.4.231

57. Jankauski M, Daniel TL, Shen IY. Asymmetries in wing inertial and aerodynamic torques contribute to steering in flying insects. Bioinspiration and Biomimetics. 2017; 12(4). https://doi.org/10.1088/17483190/aa714e PMID: 28474606 
58. Bush NE, Solla SA, Hartmann MJ. Whisking mechanics and active sensing. Current Opinion in Neurobiology. 2016; 40:178-188. https://doi.org/10.1016/j.conb.2016.08.001 PMID: 27632212

59. Wachowiak M. All in a sniff: olfaction as a model for active sensing. Neuron. 2011; 71(6):962-973. https://doi.org/10.1016/j.neuron.2011.08.030 PMID: 21943596

60. Nelson ME, Maclver MA. Sensory acquisition in active sensing systems. Journal of Comparative Physiology A. 2006; 192(6):573-586. https://doi.org/10.1007/s00359-006-0099-4 PMID: 16645885

61. Mitchinson B, Martin CJ, Grant RA, Prescott TJ. Feedback control in active sensing: Rat exploratory whisking is modulated by environmental contact. Proceedings of the Royal Society B: Biological Sciences. 2007; 274(1613):1035-1041. https://doi.org/10.1098/rspb.2006.0347 PMID: 17331893

62. Mhatre N, Sivalinghem S, Mason AC. Posture controls mechanical tuning in the black widow spider mechanosensory system. bioRxiv. 2018;484238:1-27.

63. Palka J, Malone MA, Ellison RL, Wigston DJ. Central Projections of Identified Drosophila Relation to their Time of Development Sensory Neurons in Relation to their Time of Development. Journal of Neuroscience. 1986; 6(6):1822-1830. https://doi.org/10.1523/JNEUROSCI.06-06-01822.1986 PMID: 3712012

64. Fox JL, Fairhall AL, Daniel TL. Encoding properties of haltere neurons enable motion feature detection in a biological gyroscope. Proceedings of the National Academy of Sciences. 2010; 107(8):3840-3845. https://doi.org/10.1073/pnas.0912548107

65. Dickerson BH, Fox JL, Sponberg S. Functional diversity from generic encoding in insect campaniform sensilla. Current Opinion in Physiology. 2021; 19:194-203. https://doi.org/10.1016/j.cophys.2020.11. 004

66. Marder E, Goeritz ML, Otopalik AG. Robust circuit rhythms in small circuits arise from variable circuit components and mechanisms. Current Opinion in Neurobiology. 2015; 31:156-163. https://doi.org/10. 1016/j.conb.2014.10.012 PMID: 25460072

67. Buerkle NP, Palmer SE. Ancestral neural computations constrain the evolution of novel computations in simulated color vision networks. bioRxiv. 2020;891382.

68. Furusawa $\mathrm{C}$, Irie N. Toward understanding of evolutionary constraints: experimental and theoretical approaches. Biophysical Reviews. 2020; p. 1-7. https://doi.org/10.1007/s12551-020-00708-2 PMID: 32572681

69. Dickinson $\mathrm{MH}$, Hannaford S, Palka J. The evolution of insect wings and their sensory apparatus. Brain, behavior and evolution. 1997; 50(1):13-24. https://doi.org/10.1159/000113318 PMID: 9209763

70. Blanke A. Analysis of modularity and integration suggests evolution of dragonfly wing venation mainly in response to functional demands. Journal of the Royal Society Interface. 2018; 15(145). https://doi.org/ 10.1098/rsif.2018.0277 PMID: 30158178

71. Aiello BR, Sikandar UB, Minoguchi H, Kimball KC, Hamilton CA, Kawahara AY, et al. Wing shape evolution in bombycoid moths reveals two distinct strategies for maneuverable flight. bioRxiv. 2020;358176.

72. Mountcastle AM, Daniel TL. Aerodynamic and functional consequences of wing compliance. Experiments in Fluids. 2009; 46(873).

73. Mistick EA, Mountcastle AM, Combes SA. Wing flexibility improves bumblebee flight stability. Journal of Experimental Biology. 2016; 219:3384-3390. PMID: 27638618

74. Mountcastle AM, Combes SA, Mountcastle AM. Wing flexibility enhances load-lifting capacity in bumblebees. Proceedings of the Royal Society B: Biological Sciences. 2013; 280. https://doi.org/10.1098/ rspb.2013.0531 PMID: 23536604

75. Gettrup E. Sensory mechanisms in locomotion: the campaniform sensilla of the insect wing and their function during flight. Cold Spring Harbor Symposia on Quantitative Biology. 1965; 30:615-622. https:// doi.org/10.1101/SQB.1965.030.01.059 PMID: 5219508

76. Dickinson MH. Comparison of Encoding Properties of campaniform sensilla on the fly wing. Journal of Experimental Biology. 1990; 151:245-261. https://doi.org/10.1242/jeb.151.1.245

77. Gnatzy W, Grünert U, Bender M. Campaniform sensilla of Calliphora vicina (Insecta, Diptera): I. Topography. Zoomorphology. 1987; 106:312-319. https://doi.org/10.1007/BF00312005

78. Hedrick TL, Robinson AK. Within-wingbeat damping: dynamics of continuous free-flight yaw turns in Manduca sexta. Biology Letters. 2010; 6(3):422-425. https://doi.org/10.1098/rsbl.2010.0083 PMID: 20181557

79. Dickinson $\mathrm{MH}$. Linear and nonlinear encoding properties of an identified mechanoreceptor on the fly wing measured with mechanical noise stimuli. Journal of Experimental Biology. 1990; 151:219-244. https://doi.org/10.1242/jeb.151.1.219

80. Brunton BW, Brunton SL, Proctor JL, Kutz JN. Sparse sensor placement optimization for classification. SIAM Journal on Applied Mathematics. 2016; 76(5):2099-2122. https://doi.org/10.1137/15M1036713 
81. Grant M, Boyd S. CVX: Matlab Software for Disciplined Convex Programming, version 2.1; 2014. http:// cvxr.com/cvx.

82. Grant M, Boyd S. Graph implementations for nonsmooth convex programs. In: Blondel V, Boyd S, Kimura H, editors. Recent Advances in Learning and Control. Lecture Notes in Control and Information Sciences. Springer-Verlag Limited; 2008. p. 95-110. 